\title{
Impact of the membrane viscosity on the tank-treading behavior of red blood cells
}

\author{
P. Matteoli, F. Nicoud, and S. Mendez \\ IMAG, Univ. Montpellier, CNRS, Montpellier 34095, France
}

(Dated: April 1, 2021)

\begin{abstract}
Numerical simulations supported by previously published experimental data are used to compare the impact of the internal fluid viscosity and the membrane viscosity on a tank-treading red blood cell. The method used is based on a continuum framework both for the fluid and the membrane, their interaction being ensured thanks to the immersed boundary method. The finite-strain model implemented to account for membrane viscosity assumes a free energy form that leads to an additive decomposition of the equilibrium and non-equilibrium stresses. It also assumes that the stress tensor can be separated into a deviatoric part and a hydrostatic part, which are independent. Only the deviatoric part is accounted for in this study. Both the viscosities of the cytoplasm and of the membrane yield a decrease of the tank-treading frequency, with only moderate changes in the deformation of the red blood cell. However, it is shown that the values of tank-treading frequency from the literature cannot be explained by the internal fluid viscosity only. On the opposite, adding the membrane dissipation produces a good agreement with experimental results when using acceptable values of the internal fluid and membrane viscosities. In addition, this study proposes a direct inference of the value of the membrane viscosity as a function of the shear rate from the comparison between simulations and experiments and confirms experimental results that highlighted the shear-thinning behavior of the red blood cell membrane.
\end{abstract}

\section{INTRODUCTION}

Red blood cells (RBCs) are the most common cells in blood. They are non-nucleated cells in charge of carrying oxygen to the tissues. Oxygen is fixed by the RBCs thanks to the haemoglobin contained in their cytoplasm, which is itself enclosed in a biological membrane. In humans, the RBCs are biconcave disks of characteristic sizes about $8 \mu \mathrm{m}$ in diameter and $2 \mu \mathrm{m}$ in height.

To ensure the body oxygenation, RBCs have to circulate through narrow capillaries of only a few micrometers in diameter. Moreover, in the spleen, they have to squeeze through sub-micrometer openings much smaller than their size at rest. As a consequence, RBCs need to be able to change their shape to adapt to the narrowest passages in the body. This ability is referred to as the deformability of the RBCs. In fact, two elements are important to understand this property: 1) there is an excess area of the RBCs membrane with respect to the volume of the cell, which authorizes changes of shape with mild deformation of the membrane, and 2) the membrane is a soft material able to deform. Any alteration of these elements may result in an important decrease in the RBC deformability [1]. Deformability is altered in different pathological conditions, which is sometimes used as a biomarker for diseases like malaria, sickle cell anemia or diabetes [2].

The RBC membrane is composed of a lipid bilayer and a cytoskeleton. Mechanically, the lipids of the bilayer may circulate, but the bilayer strongly resists any change of area: the lipid bilayer is thus responsible for the resistance to area change of the membrane, but also to membrane bending [3]. In this bilayer is anchored the cytoskeleton, a spectrin network that gives the elastic shear resistance to the RBC membrane [4].

Resistances to shear, area changes and bending are essential to define the RBC deformability. However, when time-varying loadings are considered, the viscosity of the RBC becomes another critical component of deformability. The viscosity of the cytoplasm has been demonstrated to control the dynamics of the RBC under shear flow $[5,6]$. On the other hand, numerous experiments have highlighted the importance of membrane viscosity in dynamic deformations [7-9].

Over the last decades, the numerical simulation of RBC dynamics has developed tremendously [10-12]. If the elastic properties of the membrane are always accounted for, membrane viscosity is very often neglected. Only a few studies include it [13-17], generally without a detailed study of its effects. The absence of membrane viscosity is sometimes invoked as an explanation for insufficient dissipation in the simulations, for instance to explain the overestimation of tank-treading frequencies of RBCs under shear [18].

One reason why membrane viscosity has been neglected in so many theoretical works is that other elements of the system may dissipate energy: the suspending fluid and the cytoplasm. [4, 16, 18, 19]. Accounting for these dissipation sources may be sufficient to reproduce qualitative trends $[5,6]$. Quantitative agreements may even be obtained in some cases: for instance, when the suspending fluid is very viscous, the membrane dissipation may be small compared to external dissipation, so that relaxation times in viscous fluids may be retrieved even neglecting membrane viscosity [16]. However, when red blood cells are suspended in a fluid with low viscosity (such as plasma or saline solutions), membrane viscosity controls the relaxation time [7, 17]. The value of membrane viscosity itself is not known precisely. Guglietta et al. have gathered values of membrane viscosity from the literature: they vary from $5.0 \times 10^{-5} \mathrm{cP}$ m to $100.0 \times 10^{-5} \mathrm{cP} \mathrm{m}$. In their recent simulations, Guglietta et al. [17] use a membrane viscosity value of $31.8 \times 10^{-5} \mathrm{cP} \mathrm{m}$ to reproduce $\mathrm{RBC}$ relaxation times. 
Experimentally, it is extremely difficult to separate different dissipation sources. In particular, internal dissipation and membrane dissipation cannot be controlled individually in an experiment. This confusion has been reinforced by some modeling works. Keller and Skalak have notably developed a theoretical model for the dynamics of RBCs in shear flow [20], which has been extended to more complex cases later [19, 21-24], in which internal viscosity and membrane viscosity act in the exact same manner, the two being combined in an effective RBC viscosity. Membrane viscosity has sometimes been used to account for the whole viscosity of the system. For example, Mancuso and Ristenpart [25] fit experimental data of RBCs stretched in a sudden contraction to extract the membrane viscosity of the RBC thanks to a model neglecting internal viscosity. Are internal viscosity and membrane viscosity equivalent? Do they contribute in the same way to the dissipation in the system? These questions are open, but numerical simulation of RBC dynamics can contribute to answering them, as we show in this study.

To compare the effect of internal and membrane viscosities, a relevant configuration is that of an RBC tanktreading in a pure shear flow, which has already been extensively studied experimentally [21, 26, 27], and numerically $[18,28-33]$. In a shear flow, when the suspending medium is viscous enough and for high enough shear stresses, the membrane circulates around the cell like the tread of a tank [20]. The RBC lengthens in the direction of the flow and adopts an almost constant angle with respect to the flow direction [21]. Tank-treading results are generally characterized in terms of $\mathrm{RBC}$ elongation, inclination angle and tank-treading frequency, measured experimentally by attaching a bead to the RBC membrane to track its circulation [34].

While simulations generally compare favorably with experiments in terms of elongation and inclination, tanktreading frequencies are overestimated in the absence of membrane viscosity in the model, as shown by Dodson and Dimitrakopoulos [18]. Because membrane viscosity contributes to internal friction in the membrane, it always opposes to the load. Moreover, it adds a delay between the load and the response. Implementation of an internal membrane dissipation is expected to slow down tank-treading motion, thus decreasing of tank-treading frequencies.

The purpose of this study is to compare the dissipative sources in an RBC and to study their impact on the tank-treading behavior. Numerical simulations with and without membrane viscosity were performed to systematically assess its effect. Because we assume membrane incompressibility, we neglect area change viscosity $[14,35]$ and only shear viscosity is accounted for in the membrane. We first introduce the numerical method and how an existing RBC simulation framework has been extended to account for membrane viscosity, with relevant validation. In this work, we build on a classical framework for predicting the dynamics of capsules and red blood cells, based on the immersed boundary method [36, 37]. In this framework, we introduce the membrane viscoelasticity following Yazdani \& Bagchi [14] and more recent works[17, 38]. However, for the sake of completeness and clarity of our approach, the numerical implementation is presented in detail in the next section. Then, the tank-treading configuration of interest is presented before detailing the results, focusing on the comparison between internal viscosity and membrane viscosity. A comparison with existing experimental results is also shown. Finally, experiments and simulation results are combined to infer the effective value of membrane shear viscosity in this configuration of tank-treading RBCs.

\section{METHODOLOGY}

\section{A. Continuum modeling of membrane viscoelasticity}

A common approach to model the membrane of an RBC suspended in a fluid is to assimilate the full membrane as a single surface and give to this infinitely thin object similar mechanical properties as the whole membrane. The membrane is thus represented as an interface between two viscous fluids, with specific rheology linking its stress state to its deformation. Generally, stress from deformation is obtained as the sum of several sub-models accounting for different mechanical properties of the membrane [11, 13, 18, 19, 28, 30]. Here, we use and extend such a continuum framework, in which an inviscid hyperelastic model accounts for the in-plane resistances in the membrane while a curvature resistance is implemented separately.

The finite-strain model implemented in this study to account for membrane viscosity is based on Simo's work [39] and assumes a free energy form that leads to an additive decomposition of the equilibrium and non-equilibrium stresses. It also assumes that the stress tensor can be separated into a deviatoric part and a hydrostatic part, which are independent. In this study, we will modify an existing inviscid model by adding purely deviatoric viscosity.

Let us first start by describing the inviscid model. Here, the in-plane elastic resistances are modeled using the Skalak hyperelastic law [40]. This law controls the membrane shear and area change resistance. In the local strain eigenbasis, it is expressed as: 


$$
\begin{aligned}
& \sigma_{11}=\frac{G_{s}}{\lambda_{1} \lambda_{2}}\left[\lambda_{1}^{2}\left(\lambda_{1}^{2}-1\right)+C\left(\lambda_{1} \lambda_{2}\right)^{2}\left[\left(\lambda_{1} \lambda_{2}\right)^{2}-1\right]\right] \\
& \sigma_{22}=\frac{G_{s}}{\lambda_{1} \lambda_{2}}\left[\lambda_{2}^{2}\left(\lambda_{2}^{2}-1\right)+C\left(\lambda_{1} \lambda_{2}\right)^{2}\left[\left(\lambda_{1} \lambda_{2}\right)^{2}-1\right]\right] \\
& \sigma_{12}=\sigma_{21}=0
\end{aligned}
$$

with $\sigma_{i j}$ the components of the Cauchy stress tensor $\boldsymbol{\sigma}$ on the surface (in $\mathrm{Pa} \mathrm{m}$ ), $\lambda_{i}$ the eigenvalues of the transformation gradient tensor $\boldsymbol{F}$ expressed as $\boldsymbol{F}=\frac{\partial x(t)}{\partial x(0)}$, with $x(t)$ the current state and $x(0)$ denotes the stressfree state. $G_{s}$ is the Skalak shear modulus (in Pa m) and $C$ the ratio between the area change modulus and the shear modulus. In this work, to ensure area incompressibility, a large value of $C$ is imposed, as is done classically for RBCs [33, 41]. This leads to an almost incompressible material, which justifies the choice of implementing membrane viscosity on the deviatoric part of the membrane deformation only. Thus, in the viscoelastic model developed in this study, the hydrostatic behavior of the membrane follows the Skalak hyperelastic law, whereas the deviatoric part is viscoelastic. We now detail how the viscoelastic model is implemented.

In the literature, the RBC viscoelasticity of the membrane is generally modeled with the Kelvin-Voigt model [8]. However, because of its versatility, and because some experimental studies point towards more complex membrane behaviors [9], we choose to implement the Zener rheological model (also named standard linear solid (SLS)) as sketched in Fig. 1. This model has also been shown to increase the stability of the numerical methods compared to a direct implementation of the Kelvin-Voigt model[14, 42]. It consists of a Maxwell model in parallel with the purely elastic model so that the stress of the two branches are added during deformation. The elastic law set in the branch of the Maxwell branch is the Hooke law, expressed as:

$$
\begin{aligned}
\sigma_{11} & =\frac{G_{s}}{1-\nu_{s}}\left[\lambda_{1}^{2}-1+\nu_{s}\left(\lambda_{2}^{2}-1\right)\right] \\
\sigma_{22} & =\frac{G_{s}}{1-\nu_{s}}\left[\lambda_{2}^{2}-1+\nu_{s}\left(\lambda_{1}^{2}-1\right)\right] \\
\sigma_{12} & =\sigma_{21}=0
\end{aligned}
$$

As a summary, the purely elastic branch follows a hyperelastic Skalak law, while in the Maxwell branch, the spring follows a Hooke law and the damper a linear viscosity law. This makes the model behave like a pure Skalak material once fully relaxed, thus ensuring that the hyperelastic properties of the model, for instance assessed by static techniques [43, 44] and validated independently [45], are conserved. However, for transient situations, the membrane will behave like the addition of the branches. The way this model is discretized and implemented actually depends on the general algorithm used to predict the dynamics of RBCs. This existing algorithm is first detailed, before presenting its modifications to include membrane viscosity.

(a)

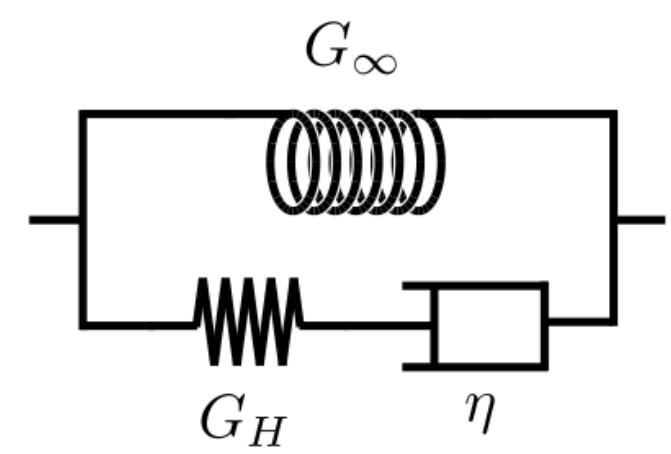

(b)

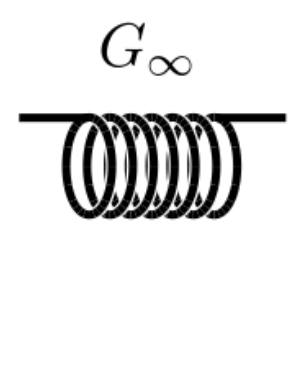

Figure 1. Rheological model of the behavior law used in this study. The curved springs represent the Skalak hyperelastic law and the straight spring the Hooke law. Viscoelastic rheological model for the deviatoric part (a) and purely hyperelastic model for the hydrostatic part (b). 


\section{B. General fluid-structure interaction algorithm}

The numerical framework used in this work is the YALES2BIO solver (https://imag.umontpellier.fr/ yales2bio/), dedicated to the simulation of RBC dynamics under flow and already used in several publications in the recent years $[5,6,19,24,45,46]$. It is based on a continuum framework both for the fluid and the membrane. The membrane is supposed to be massless and may be viewed as an interface with specific surface tension that depends on its state of deformation. The fluid and the membrane are coupled by the immersed boundary method [37]. This technique enables the communication between two meshes, one for the fluid and one for the membrane. Inside the temporal loop, the main YALES2BIO algorithm can be reduced to four main steps:

1. For a given state of deformation of the membrane and using the material behavior law, the membrane stress field is computed. Nodal forces are then calculated under the massless membrane assumption.

2. The forces at the nodes are regularized to the fluid mesh to act as a source term for the Navier-Stokes equations, representing the action of the membrane on the fluid.

3. The next step consists in solving explicitly the incompressible Navier-Stokes equations in a finite-volume framework with the membrane forcing, thanks to a pressure projection technique [47-49] for imposing fluid incompressibility. This results in an updated fluid velocity field.

4. The fluid velocity is then interpolated to the membrane vertices, with the assumption that the membrane is displaced at the same velocity as the local fluid velocity. The membrane position is then updated with an explicit Euler scheme, which provides the deformation state for the next iteration. A volume conservation algorithm is used to guarantee that the volume inside the membrane does not change during the simulation $[50,51]$. In case of a viscosity contrast between the cytoplasm and the suspending fluid, the fluid viscosity is then updated from the new membrane position, with different values inside and outside the membrane.

This algorithm is now classical and has been employed by many authors, such as [12, 14, 17, 28, 33, 36, 52, 53]. Its implementation in YALES2BIO has been presented and validated in several publications $[5,45,50,51]$ in cases without membrane viscosity. To account for membrane viscosity, the only difference lies in the first step, the calculation of membrane forces, which is now detailed.

\section{From in-plane hyperelastic forces to viscoelastic forces}

The calculation of the purely hyperelastic RBC membrane forces is based on a first-order finite-element framework that has often been used in RBC modeling [54]. It has already been validated in numerous cases $[24,50,51]$. In order to compute forces applied on the fluid from a hyperelastic membrane, $\lambda_{i}$, the eigenvalues of the transformation gradient, are first calculated for the membrane elements. Then, membrane stress is computed thanks to Eq. (1). The stress is then used to compute nodal forces using the virtual work principle as in Shrivastava and Tang's work [55]. Details about the methodology to obtain nodal forces are provided in Appendix.

Steps for implementation of viscoelastic behavior are similar, except that membrane viscous stress is a priori a function of the whole history of deformation of the membrane, so that some preliminary work is needed to express it as a function of the last time step only. Because only shear viscosity is considered here, the expression of the membrane viscoelastic behavior can be written as:

$$
\boldsymbol{\sigma}(t)=\boldsymbol{\sigma}^{d}+\sigma^{h}(t) \boldsymbol{I}=\int_{0}^{t} 2 G(t-s) \dot{\boldsymbol{\varepsilon}}^{d} d s+\sigma^{h}(t) \boldsymbol{I},
$$

with $t$ the time, $\boldsymbol{\sigma}$ the Cauchy stress tensor, $G(t-s)$ the relaxation modulus of the viscoelastic model (in Pa $\mathrm{m})$ at time $t-s, \dot{\varepsilon}^{d}$ the time derivative of the deviatoric part of the strain tensor $\varepsilon, \sigma^{h}$ the mean normal of the Cauchy stress tensor, calculated as $\sigma^{h}=\frac{\operatorname{tr}(\boldsymbol{\sigma})}{\operatorname{DIM(\boldsymbol {\sigma })}}$ and $\boldsymbol{I}$ the identity matrix.

The first hypothesis is that we are assuming additivity between the Hooke spring and the viscous damper (see Fig. 1). This leads to the following formulation of the relaxation modulus:

$$
G(t)=G_{\infty}+G_{H} e^{-\frac{t}{t_{c}}}
$$

with $G_{\infty}$ the Skalak shear modulus from the pure hyperelastic branch (in $\mathrm{Pa} \mathrm{m}$ ), $G_{H}$ the Hooke shear modulus from the viscoelastic branch (in $\mathrm{Pa} \mathrm{m}$ ), and $t_{c}$ the Maxwell characteristic time of the viscoelastic branch computed as $t_{c}=\frac{\eta}{G_{H}}$ with $\eta$ the membrane viscosity (in Pa s m). 
With the aforementioned hypotheses, Simo [39] obtained an expression for the Kirchhoff stress tensor $\boldsymbol{\tau}=$ $\operatorname{det}(\boldsymbol{F}) \boldsymbol{\sigma}$, with $\boldsymbol{\sigma}$ the Cauchy stress tensor and $\boldsymbol{F}$ the deformation gradient tensor. We will thus keep the formulation with the Kirchhoff stress tensor until the end of the derivation, which simplifies the reading and the comparison with existing literature, where the Kirchhoff tensor is classically used. Using integration by part in Eq. (3) enables to apply the derivation in time on the relaxation modulus:

$$
\boldsymbol{\tau}(t)=\boldsymbol{\tau}_{0}^{d}(t)+S Y M\left[\int_{0}^{t} \frac{\dot{G}(s)}{G_{0}} \boldsymbol{F}_{t}^{-1}(t-s) \cdot \boldsymbol{\tau}_{0}^{d}(t-s) \cdot \boldsymbol{F}_{t}(t-s) d s\right]+\tau^{h}(t) \boldsymbol{I},
$$

with $\tau_{0}^{d}$ the deviatoric part of the instantaneous Kirchhoff stress computed with $G_{0}=G(0)=G_{\infty}+G_{H}$ and $\tau^{h}(t)$ the mean normal Kirchhoff stress. Finally, $\boldsymbol{F}_{t}(t-s)$ represents the deformation gradient computed between time $t$ and time $t-s$. Also called shifter, its expression is:

$$
\boldsymbol{F}_{t_{1}}\left(t_{2}\right)=\frac{\partial x\left(t_{2}\right)}{\partial x\left(t_{1}\right)}
$$

with $x(t)$ the Lagrangian position of the solid particle at the time $t$. It is important to note that the main role of the shifter is to enable the stress sum. Indeed, for finite strains, it makes no sense to add stresses from different strain configurations. Shifters $\boldsymbol{F}_{t}(t-s)$ transport the stress at time $t-s$ to the time $t$, for every $s$ value between 0 and $t$. Now, simply combining Eq. (4) with Eq. (5) leads to:

$$
\boldsymbol{\tau}(t)=\boldsymbol{\tau}_{0}^{d}(t)-\boldsymbol{\tau}_{v i s c}(t),
$$

with $\boldsymbol{\tau}_{v i s c}(t)$ expressed as:

$$
\boldsymbol{\tau}_{v i s c}(t)=\frac{G_{H}}{t_{c} G_{0}} S Y M\left[\int_{0}^{t} \boldsymbol{F}_{t}^{-1}(t-s) \cdot \boldsymbol{\tau}_{0}^{d}(t-s) \cdot \boldsymbol{F}_{t}(t-s) e^{-\frac{s}{t_{c}}} d s\right] .
$$

From this expression, we can see that an integration between $t=0$ and $t$ is needed to compute the stress. The next step consists in modifying this expression to make it depend on times $t$ and $t-\Delta t$ only. To do so, Eq. (6) allows expressing $\boldsymbol{F}_{t}(t-s)$ as a function of $\boldsymbol{F}_{t}(t-\Delta t)$ :

$$
\boldsymbol{F}_{t}(t-s)=\boldsymbol{F}_{t-\Delta t}(t-s) \cdot \boldsymbol{F}_{t}(t-\Delta t) .
$$

Consequently, we have also:

$$
\boldsymbol{F}_{t}^{-1}(t-s)=\boldsymbol{F}_{t}^{-1}(t-\Delta t) \cdot \boldsymbol{F}_{t-\Delta t}^{-1}(t-s)
$$

By substituting Eq. (9) and Eq. (10) into Eq. (8), and by doing a variable change from $s$ to $s+\Delta t$, Eq. (8) can be split in two terms as follows:

$$
\begin{aligned}
\boldsymbol{\tau}_{v i s c}(t) & =\frac{G_{H}}{t_{c} G_{0}} S Y M\left[\boldsymbol{F}_{t}^{-1}(t-\Delta t) \cdot \int_{-\Delta t}^{0} \boldsymbol{F}_{t-\Delta t}^{-1}(t-\Delta t-s) \cdot \boldsymbol{\tau}_{0}^{d}(t-\Delta t-s) \cdot \boldsymbol{F}_{t-\Delta t}(t-\Delta t-s) e^{-\frac{s+\Delta t}{t_{c}}} d s \cdot \boldsymbol{F}_{t}(t-\Delta t)\right] \\
& +\frac{G_{H}}{t_{c} G_{0}} S Y M\left[\boldsymbol{F}_{t}^{-1}(t-\Delta t) \cdot \int_{0}^{t-\Delta t} \boldsymbol{F}_{t-\Delta t}^{-1}(t-\Delta t-s) \cdot \boldsymbol{\tau}_{0}^{d}(t-\Delta t-s) \cdot \boldsymbol{F}_{t-\Delta t}(t-\Delta t-s) e^{-\frac{s+\Delta t}{t_{c}}} d s \cdot \boldsymbol{F}_{t}(t-\Delta t)\right]
\end{aligned}
$$

We can use Eq. (8) to identify the integral from 0 to $t-\Delta t$ as a known term depending on $\tau_{v i s c}(t-\Delta t)$, which results from the previous time step. To compute the final integral term, we will assume that $\boldsymbol{\tau}_{0}^{d}$ varies linearly over one time step. Finally, we obtain the following expression that can be implemented for the computation of $\tau_{v i s c}(t)$ :

$$
\boldsymbol{\tau}_{v i s c}(t)=\alpha \frac{G_{H}}{G_{0}} \boldsymbol{\tau}_{0}^{d}(t)+\beta \frac{G_{H}}{G_{0}} \hat{\boldsymbol{\tau}}_{0}^{d}(t-\Delta t)+\hat{\boldsymbol{\tau}}^{d}(t-\Delta t) \times e^{-\frac{\Delta t}{t_{c}}}
$$

with:

$$
\left\{\begin{array}{l}
t_{c}=\frac{\eta}{G_{H}} \\
\alpha=1-\frac{t_{c}}{\Delta t}\left(1-e^{-\frac{\Delta t}{t_{c}}}\right) \\
\beta=\frac{t_{c}}{\Delta t}\left(1-e^{-\frac{\Delta t}{t_{c}}}\right)-e^{-\frac{\Delta t}{t_{c}}} \\
\hat{\boldsymbol{\tau}}_{0}^{d}(t-\Delta t)=S Y M\left[\boldsymbol{F}_{t}^{-1}(t-\Delta t) \cdot \boldsymbol{\tau}_{0}^{d}(t-\Delta t) \cdot \boldsymbol{F}_{t}(t-\Delta t)\right] \\
\hat{\boldsymbol{\tau}}^{d}(t-\Delta t)=S Y M\left[\boldsymbol{F}_{t}^{-1}(t-\Delta t) \cdot \boldsymbol{\tau}_{v i s c}(t-\Delta t) \cdot \boldsymbol{F}_{t}(t-\Delta t)\right]
\end{array}\right.
$$


Once the stress tensor computed, we use the principle of virtual work [55] to compute the associated nodal forces at the vertices of the element (see also the Appendix). The contribution of one element to the nodal force $\vec{f}_{n}$ of one of its vertices $n$ is:

$$
\vec{f}_{n}(t)=V_{\text {elem }}(t) \boldsymbol{F}_{\mathbf{0}}^{-1}(t) \boldsymbol{\sigma}(t) \vec{\varphi}_{n}
$$

with $V_{\text {elem }}$ the deformed element area and $\vec{\varphi}_{n}$ the vector of the shape function corresponding to node $n$. The final force for each node of the membrane is obtained by accumulating of the nodal forces associated with all the elements which contain that node.

\section{Model validation: Capsule in shear flow}

(a)
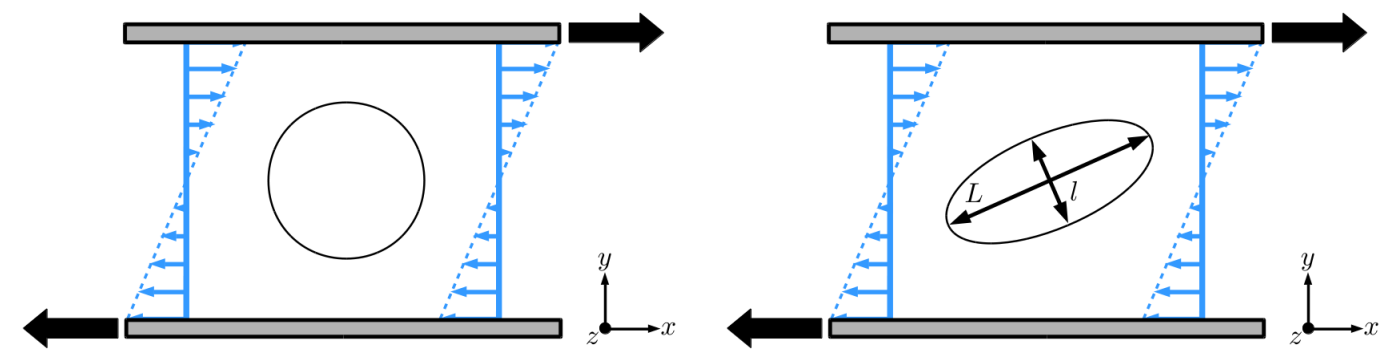

Figure 2. Representation of a capsule under shear flow. (a) Undeformed capsule at $t=0$ (b) deformed capsule at $t>0$ due to the shear flow, enabling the measurement of $D_{\text {caps }}=\frac{L-l}{L+l}$.

In order to validate the implementation of the viscoelastic model, we take advantage of recent publications reporting the dynamics of viscoelastic capsules $[17,38]$. Data for the deformation of spherical capsules in a pure shear flow (see Fig. 2). are available for different values of the membrane viscosity. In addition, the viscoelastic model used in these two studies $[17,38]$ relies on the same assumptions as those used in the present paper and described in the former section. It leads to model the capsule membrane as a Kelvin-Voigt material (case where $\left.G_{H}>>G_{\infty}\right)$. When a spherical capsule is sheared, its deformation can be characterized with a deformation index, defined as $D_{\text {caps }}=\frac{L-l}{L+l}$, with $L$ and $l$ the large and small lengths (in the shear plane), respectively, of the ellipsoid having the same inertia tensor as the capsule (see Fig. 2b).

The capsule is initially a sphere of radius $a$ described by a triangular mesh of 2890 elements. The typical edge length of this surface mesh is initially of $L_{\text {elem }} \approx \frac{a}{10}$. The capsule is deposited in a cubic fluid domain of size $20 a$, discretized by a Cartesian mesh of $200^{3}$ cubic elements, of the same size as $L_{\text {elem }}$. The domain is large enough to minimize the effects of the boundary conditions on the results [17, 38]. Two moving walls are imposed as boundary conditions at the top and bottom of the domain to impose the shear rate (see Fig. 2), so that the velocity field in the absence of capsule reads $\vec{u}=\dot{\gamma} y \overrightarrow{e_{x}}$. Periodic boundary conditions are imposed in the $x$ and $z$ directions.

The non-dimensional numbers involved in this case are the Reynolds number $R e$, the capillary number $C a$, the viscosity ratio $\lambda$ and the external Boussinesq number $B q_{\text {ext }}$. There are defined as follows:

$$
R e=\frac{\rho \dot{\gamma} a^{2}}{\mu_{e x t}} ; C a=\frac{\mu_{e x t} \dot{\gamma} a}{G_{\infty}} ; \lambda=\frac{\mu_{i n t}}{\mu_{e x t}} ; B q_{e x t}=\frac{\eta}{a \mu_{e x t}}
$$

with $\rho$ the constant density, $\mu_{i n t}$ and $\mu_{\text {ext }}$ the internal and external fluid dynamic viscosities (in Pa s), respectively, $\eta$ the membrane viscosity (in $\mathrm{Pa} \mathrm{m} \mathrm{s}$ ). All the following computations are done at $R e=0.2$ to avoid having important inertial effects while keeping the computational cost moderate, $C a=0.6$ and $\lambda=1$. Only $B q_{\text {ext }}$ is changed between the different cases. Results are displayed in Fig. 3.

Fig. 3 shows that all simulations predict the same trends regarding the effect of membrane viscosity on the dynamics of a spherical membrane: first, deformations are reduced when $B q_{\text {ext }}$ is increased. Then oscillations in the deformation appear, with a relative frequency increasing with $B q_{\text {ext }}$. The same effect has been predicted by Yazdani \& Bagchi[14]. Finally, note that the present computations are very close to the results by Guglietta et al. [17], which is the closest method to ours in terms of membrane modeling. Despite major differences in 


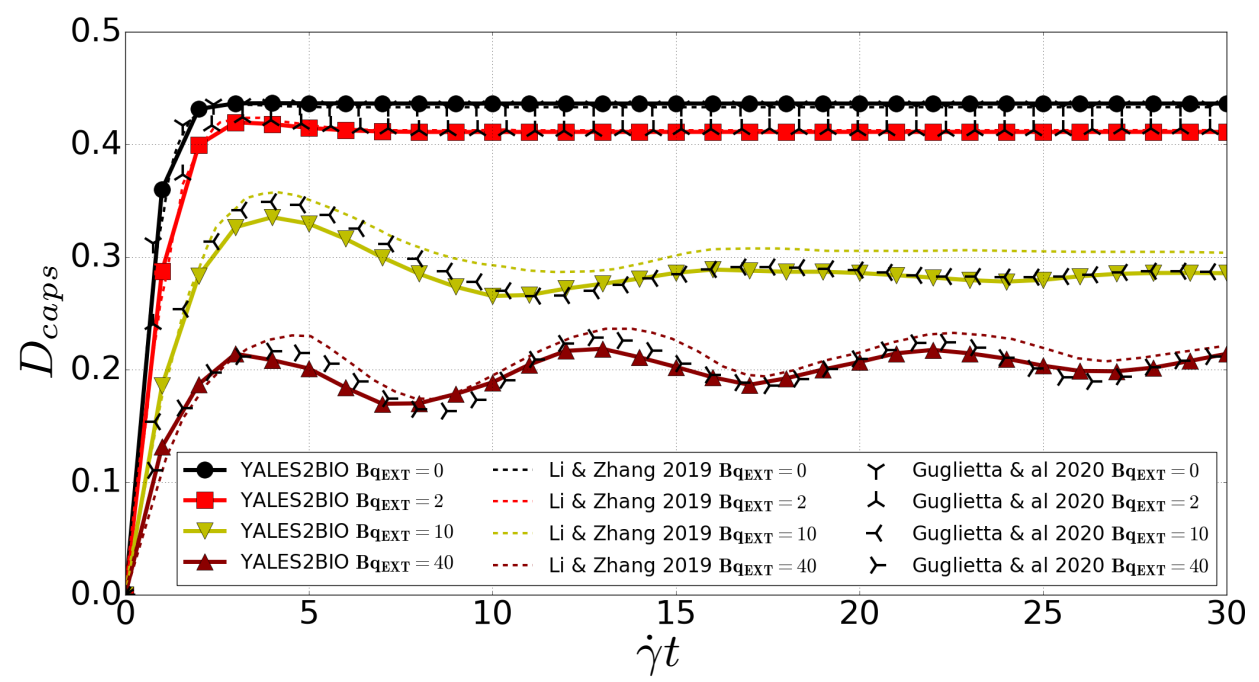

Figure 3. Deformation index as a function of non-dimensional time $\dot{\gamma} t$ for spherical capsules under shear with different $B q_{e x t}$, at $C a=0.6$ and $\lambda=1$. Comparison of present results (YALES2BIO) with those from Li and Zhang [38] and Guglietta et al. [17].

the general algorithm with the previous studies[17, 38], results align very well, so that the implementation of the viscoelastic model is validated.

\section{EFFECT OF MEMBRANE VISCOSITY ON TANK-TREADING RED BLOOD CELLS}

A. Numerical setup and operating points

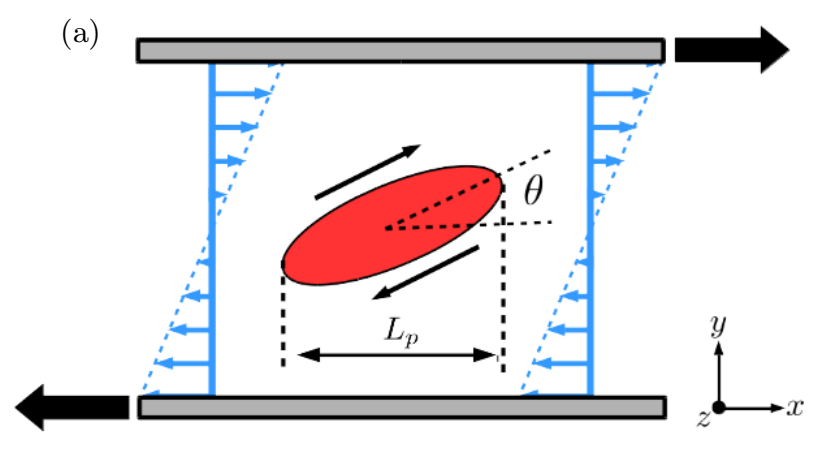

(b)

Figure 4. Representation of the tank-treading behavior. (a) Side view, enabling the measurement of the inclination angle $\theta$. Translating walls are located at the top and bottom boundaries. (b) Top view, for the measurement of the deformation index $D=\frac{L_{p}-W}{L_{p}+W}$.

Simulations of single red blood cells under pure shear are performed. The configuration is represented in Fig. 4. An external shear flow $\vec{u}=\dot{\gamma} y \overrightarrow{e_{x}}$ is imposed in a periodic domain closed by moving walls. The domain is a cube of edge $30 \mu \mathrm{m}$. The fluid inside and outside the membrane may have different values of viscosity. The $\mathrm{RBC}$ is biconcave at rest, with diameter $7.6 \mu \mathrm{m}$. Its volume is $93.9 \mathrm{\mu m}^{3}$ and its surface area $135 \mathrm{\mu m}^{2}$. Concerning the internal fluid viscosity, the literature does not provide very precise values at ambient temperature. Viscosity values between $7 \mathrm{cP}$ and $15 \mathrm{cP}$ are reported for hemoglobin solutions[56-58] for temperatures between $20^{\circ} \mathrm{C}$ and $25^{\circ} \mathrm{C}$, for a hemoglobin concentration between $330 \mathrm{~g} \mathrm{l}^{-1}$ and $360 \mathrm{~g} \mathrm{l}^{-1}$. In this work, a value close to $11 \mathrm{cP}$ will be used where dimensional results are presented (sections III C and IV).

In this study, the RBC without membrane viscosity and with fixed mechanical properties is chosen as a reference in order to highlight the impact of the lack of dissipation in the model. For every simulated RBC 
presented in this study, the Skalak shear modulus value is fixed to $G_{\infty}=2.5 \times 10^{-6} \mathrm{~Pa} \mathrm{~m}[6,59]$. The bending modulus $E_{b}$ is fixed to $6.0 \times 10^{-19} \mathrm{~J}$, which allows to slightly extend the upper bound in $C a$ for which stable results can be obtained without membrane viscosity. This value is higher than the classically measured value (around $2.0 \times 10^{-19} \mathrm{~J}$ ), but such values increased bending modulus are regularly used in the literature[28, 52, 53]. In the capillary range used in this study, we found no impact of $E_{b}$ on the deformation index $D$ and the tanktreading frequencies. Using those parameter values, the initial biconcave shape is obtained by defining the stress-free shape of the RBC as an oblate ellipsoid[28] with an axis ratio of 0.9 and the same surface area as the $\mathrm{RBC}$ at equilibrium and by deflating the stress-free shape to the RBC volume of $93.9 \mathrm{\mu m}^{3}$ in order to obtain the equilibrium shape $[15,28]$. Finally, regarding the viscoelastic RBCs, the viscoelastic branch shear modulus is set to $G_{H}=50 \times G_{\infty}$, so that the model behaves almost like a Kelvin-Voigt model, which is often done in numerical simulations $[8,15,17]$.

The RBC membrane is discretized with 3919 triangles with a characteristic element size of $0.3 \mu \mathrm{m}$. To ensure the accuracy of the immersed boundary method, the characteristic size of the fluid mesh is the same [60]. The $\mathrm{RBC}$, initially at rest, is subjected to the shear flow at $t=0$. After a brief transient phase, the shape of the $\mathrm{RBC}$ and its inclination angle $\theta$ reach a quasi-steady state, with small oscillations due to the circulation of the stress-free shape of the membrane [21, 24]. The deformation is characterized with a deformation index calculated from the top view (in the $(x, z)$ plane) $D=\frac{L_{p}-W}{L_{p}+W}$ (see Fig. 4 ). The tank-treading frequency $f_{T T}$ (in $\left.\mathrm{s}^{-1}\right)$ is also extracted. Simulations are performed for a total of $150 t^{*}$ with $t^{*}=\dot{\gamma} t$ the non-dimensional time. $D, \theta$ and $f_{T T}$ are computed over the last $120 t^{*}$.

In this study, we vary the external shear rate $\dot{\gamma}$, the external viscosity $\mu_{\text {ext }}$, the internal viscosity $\mu_{\text {int }}$ and the membrane viscosity $\eta$. The other parameters of the model (geometry, mechanical properties) are considered fixed and are representative of an average value for RBCs. We thus ignore natural variability or the effect of age on these RBC characteristics $[59,61]$. On the other hand, a systematic study of the effects of membrane viscosity and internal viscosity is performed.

Parameters for the simulations will be expressed in terms of non-dimensional numbers. As for the case with capsules, the non-dimensional numbers of the problem are the Reynolds number $R e$, the capillary number $C a$, the viscosity ratio $\lambda$ and a Boussinesq number characterizing the membrane viscosity. Concerning RBCs, we chose to use the internal Boussinesq number $B q_{i n t}=\frac{\eta}{a \mu_{i n t}}$, with $a=2.82 \mu \mathrm{m}$ the radius of a sphere of the same volume as that of the RBC, $93.9 \mu^{3}$. This non-dimensional number compares the membrane viscosity with the internal fluid viscosity. Here, it is preferred to the external Boussinesq number, as $B q_{\text {int }}$ characterizes the RBC only, independently of its environment. As for capsules, the Reynolds number was fixed to a small value of 0.2 to shorten the computation times without introducing fluid inertia effects.

\begin{tabular}{|c|c|c|c|c|}
\hline Name & $\lambda$ & $B q_{\text {int }}$ & $\mathrm{Ca}$ & Figure \\
\hline \multirow[t]{2}{*}{ L02B0 } & 0.2 & 0.0 & $0.3,0.9,2.0,3.6$ & Fig. 7(a,b) \\
\hline & & & $0.3,1.0,2.0$ & Fig. $5(\mathrm{a}-\mathrm{c})$ \\
\hline \multirow[t]{2}{*}{ L05B0 } & 0.5 & 0.0 & $0.3,0.8,1.2,2.0,3.6$ & Fig. 7(a) \\
\hline & & & $0.3,1.0,2.0$ & Fig. 5(a,c) \\
\hline \multirow[t]{2}{*}{ L1B0 } & 1.0 & 0.0 & $0.3,0.8,1.2,2.0,3.6$ & Fig. 7(a) \\
\hline & & & $0.3,1.0,2.0$ & Fig. 5(a-c) \\
\hline \multirow[t]{2}{*}{ L02B1 } & 0.2 & 1.0 & $0.3,0.9,2.0,4.3$ & Fig. 7(b) \\
\hline & & & $0.3,1.0,1.5,2.0$ & Fig. 5(a-c) \\
\hline \multirow[t]{2}{*}{ L02B3 } & 0.2 & 3.0 & $0.3,0.9,2.0,4.3$ & Fig. 7(b) \\
\hline & & & $0.3,1.0,1.5,2.0$ & Fig. 5(a-c) \\
\hline L02B5 & 0.2 & 5.0 & $0.3,1.0,1.5,2.0$ & Fig. $5(\mathrm{a}-\mathrm{c})$ \\
\hline L084B0 & 0.84 & 0.0 & $0.3,0.5,1.0,1.9$ & Fig. 7(c) \\
\hline L084B1 & 0.84 & 1.0 & $0.3,0.5,1.0,1.9$ & Fig. 7(c) \\
\hline L084B3 & 0.84 & 3.0 & $0.3,0.5,1.0,1.9$ & Fig. 7(c) \\
\hline
\end{tabular}

Table I. Recap of the simulations used in the non-dimensional and dimensional studies.

The purpose of this study is to highlight the impact of both $\lambda$ and $B q_{\text {int }}$ on the dynamics of tank-treading $\mathrm{RBCs}$, for different values of $C a$. Increasing either $\lambda$ or $B q_{i n t}$ is expected to produce similar effects associated with an increase of the total dissipation in the RBC. A recap of the computations done in this study can be found in Tab. I.

The numerical results are presented in two different ways: first, in a non-dimensional framework to present the impact of the membrane viscosity on the characteristics of a tank-treading RBC and to highlight the difference between the effects of internal and membrane viscosities. Then, results are presented in a dimensional way to enable the comparison with experimental data on the tank-treading frequency. 


\section{B. Non-dimensional study}

In this non-dimensional study, results are presented in terms of inclination angle $\theta$, deformation index $D$ and non-dimensional frequency[14, 33] $f_{T T}^{*}=\frac{4 \pi f_{T T}}{\dot{\gamma}}$, as a function of $C a$. We will highlight how the characteristics of tank-treading depend on $\lambda$ and $B q_{i n t}$, i.e. of the internal viscosity and the membrane viscosity. To do so, the following ranges of non-dimensional parameters were considered. The range of $C a$ is from 0.3 to 2.0, in particular to avoid the low- $\mathrm{Ca}$ region, where tank-treading is not stable and out-of-plane motions and deformations are possible [19, 62]. In addition, high values of the capillary number yield sharp shapes of RBCs [34] which may be unstable at the tip. Concerning the range of $\lambda$, it is well known that the tank-treading behavior is not stable anymore for $\lambda$ values greater than 2.5-3.0 [4-6, 19]. In addition, experimental studies of the tank-treading behavior are generally performed in dextran solutions, with a high external viscosity [27]. As a consequence, the range of $\lambda$ was chosen to be between 0.2 and 1.0. Finally, $B q_{\text {int }}$ values were chosen between 0 (no membrane viscosity) and 5.0, for which the tank-treading frequency is largely decreased (see further).

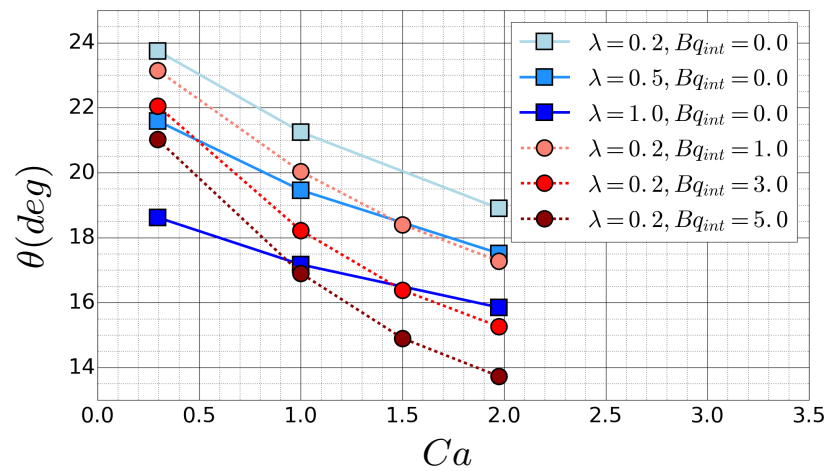

(a)

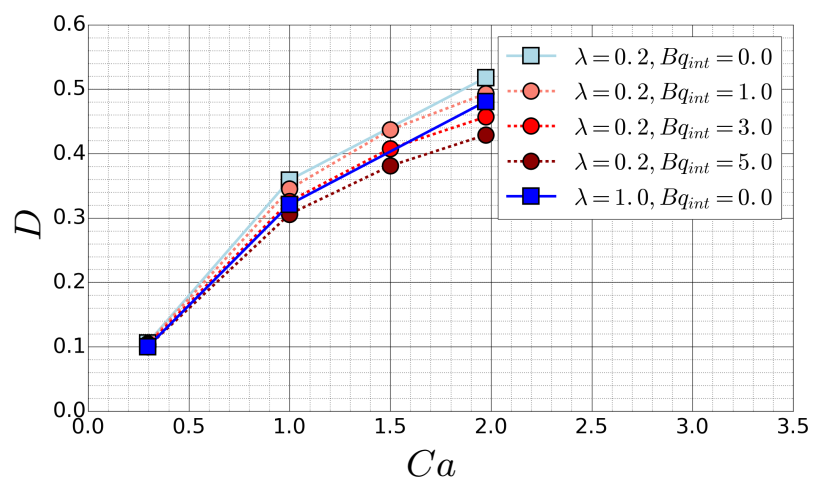

(b)

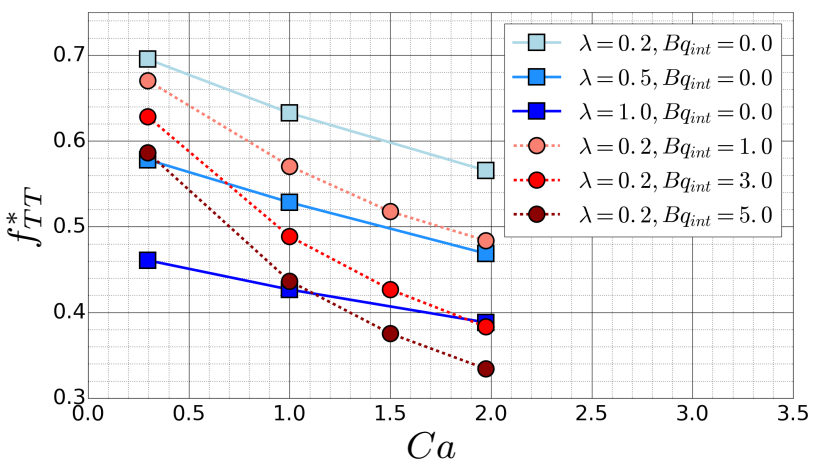

(c)

Figure 5. Impact of the internal viscosity and the membrane viscosity on the tank-treading characteristics of a single $\mathrm{RBC}$ in shear flow as a function of $C a$. (a) Inclination of the RBC, (b) deformation index and (c) the non-dimensional frequency. Only chosen data are represented on (b) to improve clarity.

Computational results from the L02B0, L05B0, L1B0, L02B1, L02B3 and L02B5 series are displayed in Fig. 5. First, the deformation index is not very sensitive to the value of $\lambda$ and $B q_{\text {int }}$ (see Fig. 5b), in particular at low values of $C a$. At higher values of $C a$, both $\lambda$ and $B q_{\text {int }}$ have the same effect of slightly decreasing the 
deformation. However, Fig. 5(a,c) show a different effect of $\lambda$ and $B q_{\text {int }}$ on the tank-treading frequency and on the inclination angle. In particular, differences are more pronounced on the frequency (Fig. 5c). Fig. 5(c) first shows that the non-dimensional frequency decreases when $C a$ increases: the tank-treading frequency is not linear with respect to $C a$ (a linear dependency would correspond to constant $f_{T T}^{*}$ in Fig. 5c). This has also been obtained in simulations without membrane viscosity [33]. Fig. 5(c) also highlights the differences between an increase in internal viscosity or in membrane viscosity. While both lead to a decrease of $f_{T T}^{*}$, this decrease is rather uniform when increasing $\lambda$, but not increasing $B q_{i n t}$. Membrane viscosity has a mild effect at low shear stresses, but its influence increases with shear stress. The same comments can be made on the effect of $\lambda$ and $B q_{i n t}$ on the inclination angle (Fig. 5a). Finally, those tendencies are also confirmed by works on the capsule tank treading [14] [38]. Indeed, even if works on capsule tank treading and impact of the membrane viscosity on this behavior are done in a larger range of $B q$, results in the same range of $B q$ as in this study show similar tendencies of the impact of viscosity on the deformation index, on the angle [14, 38] and on the frequencies [14].
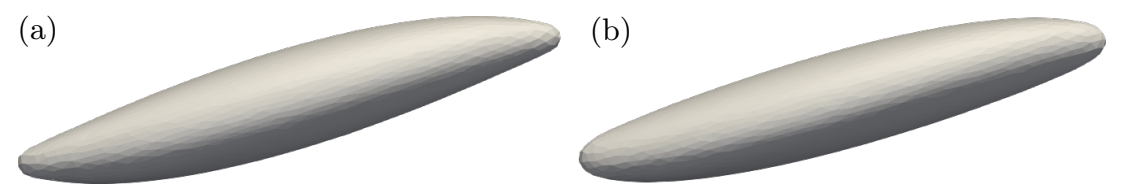

Figure 6. RBC shape comparison at $C a=2$ for L1B0 (a) and L02B3 (b), corresponding to $\lambda=1, B q_{\text {int }}=0$ and $\lambda=0.2$, $B q_{\text {int }}=3$, respectively. Corresponding values of $D$ are 0.48 for L1B0 and 0.46 for L02B3. Corresponding $f_{T T}^{*}$ is about 0.38 for both. Shape differences can mostly be seen on the extremities of the RBC. Different combinations of $\lambda$ and $B q_{\text {int }}$ yield almost identical cells, but for a given value of $C a$ only.

From the modeling point of view, the consequence is that membrane viscosity and internal viscosity are not interchangeable. For instance, for tank-treading, one cannot properly mimic membrane viscosity by increasing internal viscosity, contrary to what may be suggested in low-order models and in specific situations [20, 25]. As a matter of fact, it is possible to obtain similar tank-treading frequencies from two different RBCs, as illustrated by comparing L1B0 $\left(\lambda=1, B q_{i n t}=0\right)$ and L02B3 $\left(\lambda=0.2, B q_{i n t}=3\right)$ at $C a=2.0$. Even the shapes are pretty similar, despite little differences that can be seen on Fig. 6 . However, the similarity is only possible for a given $\mathrm{Ca}$ and does not hold for a whole range of $\mathrm{Ca}$ (see how the L1B0 and L02B3 trajectories differ with varying $C a$ in Fig. $5 \mathrm{c}$ ). In other words, it is not possible to infer internal viscosity value or membrane viscosity value using only one $\mathrm{RBC}$ and only one value of $C a$.

The difference between the impacts of the internal viscosity and of the membrane viscosity has been highlighted in the non-dimensional analysis. Now, it is interesting to study the consequences of those differences in comparison with experimental data in a dimensional framework. To do so, we will make the non-dimensional data (Tab. I) dimensional to match experimental operating conditions.

\section{Dimensional study}

As previously explained, tank-treading is classically characterized by three main parameters: the tanktreading frequency $f_{T T}$, the deformation of the RBC represented by its deformation index $D=\frac{L_{p}-W}{L_{p}+W}$ and the inclination angle between the RBC and the flow direction $\theta$. Here we specifically compare simulation results with experiments in terms of frequency, for which differences between the impacts of internal and membrane viscosities have been emphasized, and for which data is available in the literature. Experimental data for tank-treading frequencies are extracted from Fischer's work [27]. In this study, tank-treading experiments are performed with three different blood samples, for multiple external fluid viscosities and different shear rates, at a room temperature of $23^{\circ} \mathrm{C}$. We selected cases with external fluid viscosity of $12.9 \mathrm{cP}$ and $53.9 \mathrm{cP}$. Assuming that the internal viscosity of the $\mathrm{RBC}$ is $\mu_{i n t}=10.78 \mathrm{cP}$, which is an acceptable value at ambient temperature (see section III A), such values of external viscosity correspond to $\lambda=0.84$ and $\lambda=0.2$, respectively. Results are presented in Fig. $7(\mathrm{a}-\mathrm{c})$. Experimental results are presented in terms of average and error bars from Fischer's data [27]. Blood samples used in this study come from three different donors. As experimental data are dispersed both in terms of tank-treading frequency and shear rate at which it is measured, mean values and standard deviation were computed for each cloud of data and reported in Fig. 7.

First, it is seen in all subfigures that for the baseline cases without membrane viscosity, the tank-treading frequency is overestimated with respect to the experimental data. This is consistent with existing results from the literature [18]. Then, an increase either in the internal viscosity or in the membrane viscosity leads to a decrease in the tank-treading frequencies, as shown for the case with $\mu_{\text {ext }}=53.9 \mathrm{cP}$ (Fig. 7a,b): additional dissipation in the RBC slows down the membrane circulation, whatever the source for this extra dissipation. It 


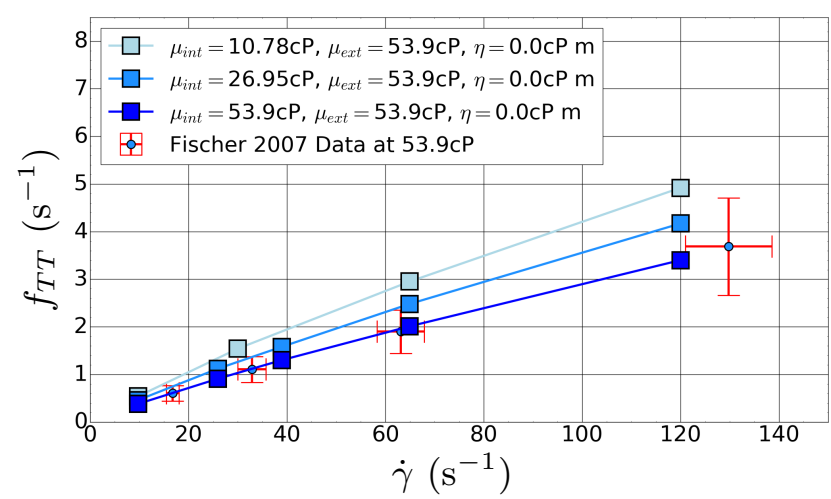

(a)

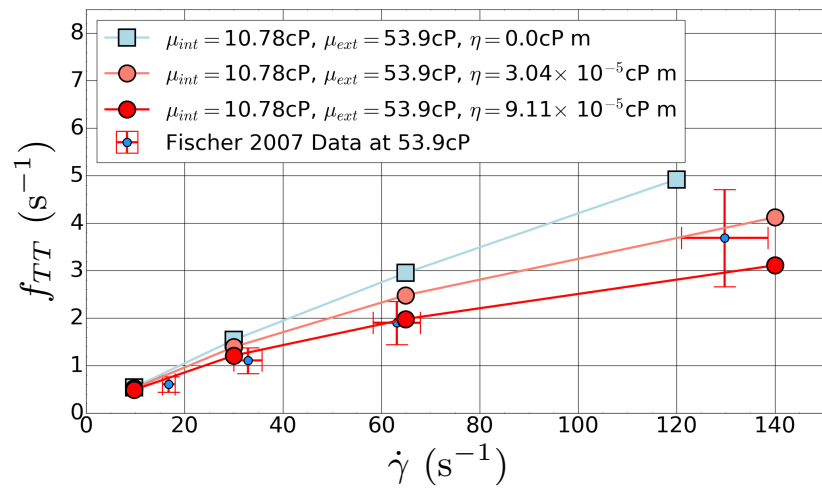

(b)

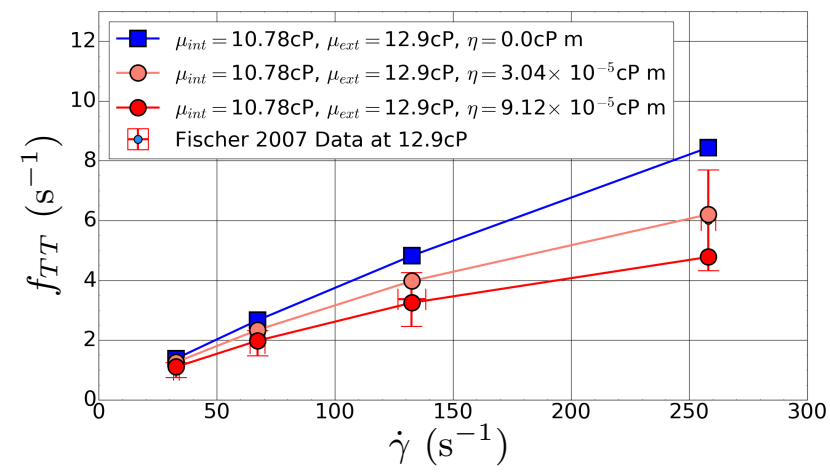

(c)

Figure 7. Comparison between computational and experimental results. (a) Impact of the internal fluid viscosity on tank-treading frequencies for an external viscosity of $\mu_{\text {ext }}=53.9 \mathrm{cP}$. (b) and (c) Impact of the membrane viscosity on frequencies for two different external viscosities $\left(\mu_{\text {ext }}=53.9 \mathrm{cP}(\mathrm{b})\right.$ and $\left.\mu_{e x t}=12.9 \mathrm{cP}(\mathrm{c})\right)$. Error bars represent two times the standard deviation observed experimentally.

is interesting to comment the value of the internal viscosity and membrane viscosity needed to get results close to the experiments: Fig. 7(a) shows that, in order to obtain simulation results comparable with experimental measurements, we have to increase the internal viscosity up to a value of $53.9 \mathrm{cP}$. This value is typically five times the expected value of the internal viscosity, which is unrealistic: even if some uncertainties exist on the value of internal viscosity, this value is not consistent with the expected range. In addition, if such a high value of internal viscosity is used with $\mu_{\text {ext }}=12.9 \mathrm{cP}$, tank-treading is not obtained anymore as the viscosity contrast is too high $[5,6]$. As a consequence, we do not present results increasing the internal viscosity with $\mu_{\text {ext }}=12.9$ cP. Indeed, results at $\mu_{\text {ext }}=12.9 \mathrm{cP}$ Fig. 7 (c) shows that the same range of membrane viscosity yields favorable comparison with experiments. Consequently, membrane viscosity seems indispensable to explain the behavior RBCs during tank-treading.

Fig. 7(b,c) allow detailing the impact of the membrane viscosity on tank-treading frequency. Interestingly, the effect of membrane viscosity is not uniform with different shear rates, as reported in the non-dimensional analysis. At low shear rates, the tank-treading frequencies seem relatively unaffected by membrane viscosity. The effect of membrane viscosity is all the more important that shear rate (and thus tank-treading frequency) increases. This is true for both $\mu_{\text {ext }}=53.9 \mathrm{cP}$ (Fig. 7(b)) and $\mu_{\text {ext }}=12.9 \mathrm{cP}$ (Fig. 7(c)).

In the series of simulations presented in Fig. 7, we have fixed the ratio between the membrane viscosity 
and the internal viscosity (in other terms, the internal Boussinesq number $B q_{\text {int }}$ ) to three different values: $B q_{\text {int }}=0.0,1.0$ and 3.0. It leads to three different $\eta$ values, $0.0 \mathrm{cP} \mathrm{m}, 3.04 \times 10^{-5} \mathrm{cP} \mathrm{m}$ and $9.11 \times 10^{-5} \mathrm{cP}$ $\mathrm{m}$, respectively. Clearly, $\eta=3.04 \times 10^{-5} \mathrm{cP} \mathrm{m}$ and $\eta=9.11 \times 10^{-5} \mathrm{cP} \mathrm{m}$ yield better results than $\eta=0.0 \mathrm{cP}$ $\mathrm{m}$, and this range of membrane viscosity yields fair comparisons with the experiment. However, the evolution with shear rate of the relative impact of the membrane viscosity on the results makes results at $\eta=3.04 \times 10^{-5}$ cP m (pink curve in Fig. $7(\mathrm{~b}, \mathrm{c})$ ) better at high shear rates and results at $\eta=9.11 \times 10^{-5} \mathrm{cP} \mathrm{m}$ (red curve in Fig. $7(\mathrm{~b}, \mathrm{c})$ ) better at low shear rates. Note that this statement seems independent of the value of external viscosity.

To conclude, even if imposing a large value of internal viscosity can reproduce experimental tank-treading frequencies in some cases, membrane viscosity is the only source for dissipation enabling a consistent improvement of the agreement between simulations and experiments for a large range of operating conditions. Indeed, the values of membrane viscosity that yield a satisfactory comparison with experiments are similar to those extracted by Tran-Son Tay [35]. However, they are higher than the values used by Guglietta et al. [17] to predict relaxation experiments. However, a constant membrane viscosity does not lead to a perfect agreement between experiments and simulations. This result suggests that a viscoelastic modeling of the membrane with a constant shear viscosity is not enough, and that a more complex membrane viscosity behavior should be accounted for. The idea of a shear-thinning behavior for the RBC membrane is not new. Chien et al. [63] reported a shear-thinning behavior of the membrane from micropipette experiments. Tran-Son Tay et al. studied the shear-thinning behavior of the RBC's membrane in the case of tank-treading RBCs and studied differences of viscosity for young and old cells [35] using the analytical model from Keller and Skalak [20]. The variation of the apparent membrane viscosity with the shear is discussed from our results in the newt section.

\section{SHEAR-THINNING MODEL}

Results obtained with a constant membrane viscosity suggest that higher values of membrane viscosity are needed for low shear rates to reproduce experimental tank-treading frequencies. This is in line with previous experimental works pointing out the shear-thinning behavior of the membrane [35, 63].

In this section, the value of membrane viscosity is inferred from the comparison between numerical predictions of the tank-treading frequency and experimental data[27] already reported in Fig. 7, at different values of the external viscosity. We use a unique red blood cells with fixed characteristics except for the membrane viscosity. For each experimental cloud (at approximatively 15, 35, 65, 130 and $260 \mathrm{~s}^{-1}$, see Fig. 7), we use the ensemble average of the shear rate as a reference. For that shear rate, simulations with different values of the membrane viscosity are performed and the tank-treading frequency is compared to the ensemble average of each cloud (this data is plotted as blue dots in Fig. 7). By dichotomy, we converge to an inferred value of membrane viscosity. The value was considered to be accurate if the difference between the numerical and the experimental frequencies was less than $5 \%$. The results of this process in terms of frequencies are displayed in Fig. 8(a,b).

Fig. 8(c) shows the inferred values of membrane viscosity as a function of the shear rate, for $\mu_{\text {ext }}=12.9 \mathrm{cP}$ and $\mu_{\text {ext }}=53.9 \mathrm{cP}$. In addition to the value of membrane viscosity yielding a good comparison with the average frequency in the experiment, we use simulations to extract a dispersion on the values of membrane viscosity (displayed in bars in Fig. 8c) from the dispersion of the experimental data. Experimental dispersion is measured using the standard deviation of the data $\Delta f_{T T}$. In simulations, we calculate how a change in membrane viscosity changes the resulting tank-treading frequency: this means calculating $\partial f_{T T} / \partial \eta$ at the inferred membrane viscosity. We define the dispersion in membrane viscosity as $\Delta \eta=\Delta f_{T T} \times \partial \eta / \partial f_{T T}$.

Fig. 8(c) leads to confirm the shear thinning behavior of the membrane, with a factor of 10 between maximum and minimum viscosity values. Moreover, error bars are bigger as the shear rate decrease. It leads to highlight that the membrane viscosity seems to be a more sensitive parameter as the shear rate increase. Finally, Fig. 8(c) also shows that the external fluid viscosity does not seem to have a major impact on the shear thinning curve. Note that at low shear rates, a large change in the membrane viscosity is needed to change the resulting tank-treading frequency, which may explain the largest differences seen at $\dot{\gamma} \approx 30 \mathrm{~s}^{-1}$.

Tran-Son Tay [35] made a similar effort to extract membrane viscosity from experimental data and a model. The corresponding results are also displayed in Fig. 8(c). Even if differences exist between Tran-Son Tay's results and our results, the trends are similar, even using very different methods to obtain those shear-thinning curves. In comparison, values of membrane viscosities computed with YALES2BIO seem closer to the values for young cells, at high shear rates. The values of membrane viscosity of Fig. 8(c) also seem to be consistent with the one used by Guglietta et al. [17] in their simulations of $\mathrm{RBC}$ relaxation $\left(\eta=3.18 \times 10^{-4} \mathrm{cP} \mathrm{m}\right)$, which could then be interpreted as a value relevant to slow deformations of the membrane. Finally, using values obtained thanks to the tank-treading behavior in order to compute relaxation time, it leads to characteristic times between 0.1 $\mathrm{s}$ and $0.01 \mathrm{~s}$, which is in accordance with literature values [17]. 


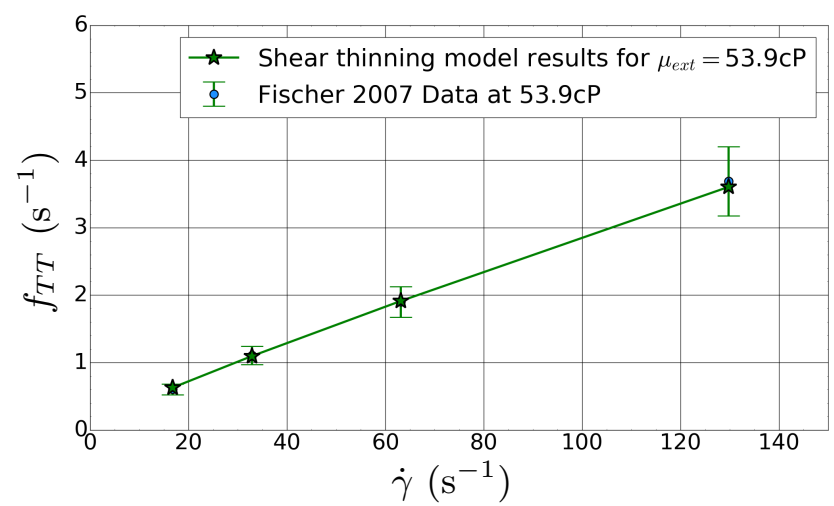

(a)

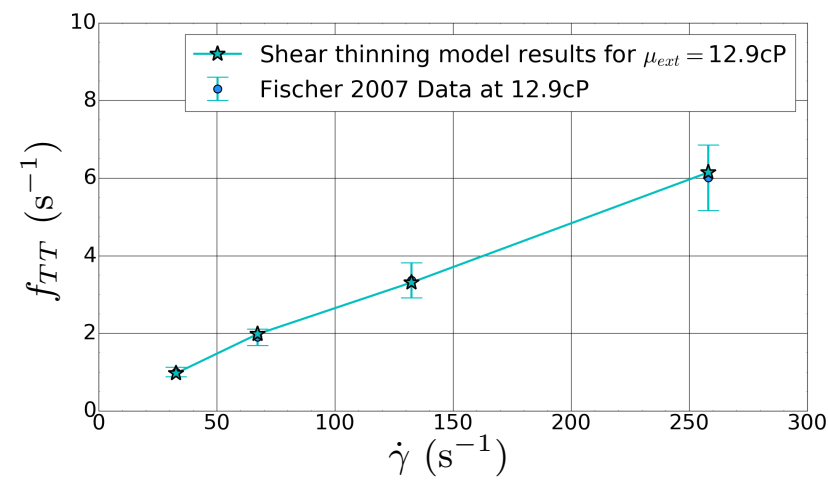

(b)

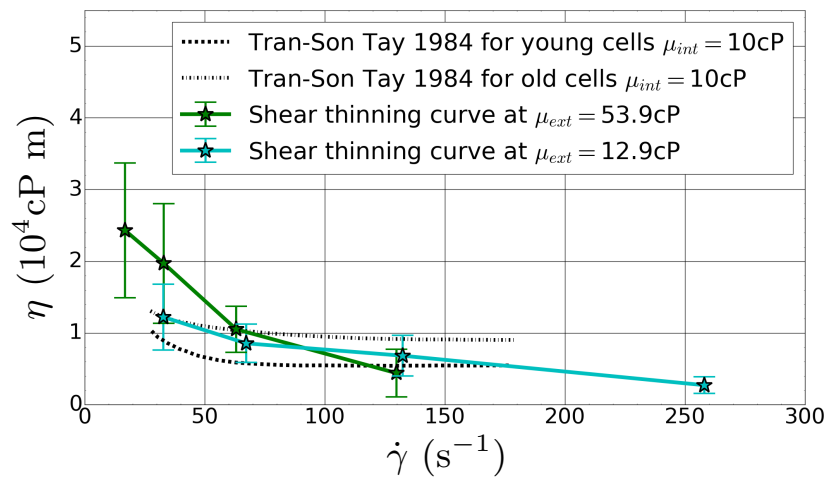

(c)

Figure 8. Comparison between the experimental frequencies[27] and the simulation frequencies obtained from the inferred membrane viscosity values. (a) Comparison with data at $\mu_{\text {ext }}=53.9 \mathrm{cP}$, (b) comparison with data at $\mu_{\text {ext }}=12.9 \mathrm{cP}$. (c) Inferred shear-thinning behavior of the membrane viscosity; values from Tran-Son Tay[35] are included for comparison. Bars in (a) and (b) represent the standard deviation of the experimental data. Bars in (c) represent the range of value of membrane viscosity in order to reproduce the dispersion of the experimental frequencies.

\section{CONCLUSION AND OUTCOMES}

Characterizing dissipation in the RBC dynamics has always been problematic, because multiple sources are present and it is difficult to stratify their effects. For the first time, simulations are presented to specifically identify the respective roles of internal and membrane viscosity in a tank-treading RBC, using comparisons with experiments. This study highlights the fact that, because the impact on the RBC dynamics are different as a function of the dissipation sources, it is difficult to aggregate these two phenomena, without more knowledge on each impact separately. This study also shows that, to reproduce experimental tank-treading frequencies, increasing only the internal viscosity leads to values out of the accepted range in the literature. Moreover, depending of the viscosity ratio, some configurations are impossible to reproduce only by increasing, even artificially, the internal viscosity (see Fig. 7c). On the other hand, the addition of membrane dissipation allows reproducing literature results by using realistic membrane viscosity values. Even if a constant viscosity value enables to improve the comparison, reproducing experimental mean frequencies could be done only by using a shear-thinning model. This study confirms experimental results that highlight the shear-thinning behavior of 
the RBC membrane, with simulations that fully account the fluid-structure interactions, not simplified models [20]. Moreover, the computed membrane viscosity values are consistent with values found by Tran-Son Tay[35] as well as in other relaxation studies $[17,64,65]$. The next step is to challenge this shear-thinning membrane model in other flow situations in order to establish its generality. In particular, assessing the effect of membrane viscosity on the behavior of a single RBC in external media with a dynamic viscosity similar to that of water or plasma (i.e. for viscosity ratios of the order of 5.0) is an interesting perspective of this work.

\section{ACKNOWLEDGEMENT}

This work/project was publicly funded through ANR (the French National Research Agency) under the 'Investissements d'avenir' programme with the reference ANR-16-IDEX-0006. We also thank Horiba Medical for their support of the project. Simulations with YALES2BIO were performed using HPC resources from GENCI-CINES (Grants A0060307194 and A0080307194) and from the platform MESO@LR.

\section{APPENDIX: FROM ELEMENT STRESS TO NODAL FORCES}

In YALES2BIO, a first-order finite-element method is used. The computation of the nodal forces from the stress in the element is detailed in what follows. We use only triangular elements, which are assumed to remain flat during the deformation of the membrane. Thus, in the element basis, deformations are two-dimensional, and each displacement can be split into $X$ and $Y$ components, respectively horizontal and vertical axis in the element basis. Noting $u_{1}, u_{2}, u_{3}$ and $v_{1}, v_{2}, v_{3}$ the $X$ and $Y$ displacements, respectively, of the nodes 1,2,3 of any triangular element, and assuming linear interpolation between the three nodes, displacements on the element can be written as:

$$
\begin{aligned}
& u=N_{1} u_{1}+N_{2} u_{2}+N_{3} u_{3}, \\
& v=N_{1} v_{1}+N_{2} v_{2}+N_{3} v_{3},
\end{aligned}
$$

with $N_{1}, N_{2}, N_{3}$ linear shape functions of the element, that can be computed as:

$$
\begin{aligned}
& N_{1}=\frac{\left(y_{2}-y_{3}\right) x+\left(x_{3}-x_{2}\right) y+\left(x_{2} y_{3}-x_{3} y_{2}\right)}{2 A_{0}}=\frac{a_{1} x+b_{1} y+c_{1}}{2 A_{0}}, \\
& N_{2}=\frac{\left(y_{3}-y_{1}\right) x+\left(x_{1}-x_{3}\right) y+\left(x_{3} y_{1}-x_{1} y_{3}\right)}{2 A_{0}}=\frac{a_{2} x+b_{2} y+c_{2}}{2 A_{0}}, \\
& N_{3}=\frac{\left(y_{1}-y_{2}\right) x+\left(x_{2}-x_{1}\right) y+\left(x_{1} y_{2}-x_{2} y_{1}\right)}{2 A_{0}}=\frac{a_{3} x+b_{3} y+c_{3}}{2 A_{0}},
\end{aligned}
$$

with $A_{0}$ the element area at $\mathrm{t}=0 . a_{i}, b_{i}$ and $c_{i}$ are the coefficients of the shape functions, only determined by the shape of the element at rest. Since displacements can be fully expressed as a function of known parameters following Eq. (17), the displacement can be used to express the two-dimensional transformation gradient $\boldsymbol{F}=$ $\frac{\partial x(t)}{\partial x(0)}$. It can also be expressed as $F_{i j}=\delta_{i j}+\frac{\partial u_{i}}{\partial x_{j}}$. Using Eq. (16) and Eq. (17), $\boldsymbol{F}$ becomes:

$$
\boldsymbol{F}=\frac{1}{2 A_{0}}\left(\begin{array}{cc}
2 A_{0}+\sum_{i=0}^{3} a_{i} u_{i} & \sum_{i=0}^{3} b_{i} u_{i} \\
\sum_{i=0}^{3} a_{i} v_{i} & 2 A_{0}+\sum_{i=0}^{3} b_{i} v_{i}
\end{array}\right)
$$

Eq. (18) will be used when writing the principle of virtual work in order to obtain the nodal forces. Because we are neglecting inertial effects, equilibrium of forces and moment equations can be written as:

$$
\operatorname{div}(\boldsymbol{\sigma})=\overrightarrow{0} ; \boldsymbol{\sigma}^{T}=\boldsymbol{\sigma}
$$

The virtual work principle is just a weak formulation of the following equilibrium equations. It is obtained by integrating stress Cauchy tensor $\boldsymbol{\sigma}$ contracted to a virtual arbitrary displacement vector $\overrightarrow{\delta u}$, with $\delta u$ and $\delta v$ its components, over the element's area. This leads to this formulation:

$$
\int_{S} d i v(\boldsymbol{\sigma}) \cdot \overrightarrow{\delta u} d s=0
$$


Using the divergence theorem, it leads to:

$$
\int_{S} \boldsymbol{\sigma}: \operatorname{grad}(\overrightarrow{\delta u}) d s-\int_{\Omega S} \vec{S} \cdot \overrightarrow{\delta u} d l=0
$$

with $\Omega S$ the element's boundary, $\overrightarrow{\delta u}$ the virtual displacement vector, $d l$ the integration variable representing an infinitesimal part of the element's boundary and $\vec{S}$ the traction vector $\vec{S}=\boldsymbol{\sigma} \vec{n}$, with $\vec{n}$ the outward unit normal vector of the element's boundary. The gradient term can be written as:

$$
\boldsymbol{g r a d}(\overrightarrow{\delta u})=\frac{\partial \delta u}{\partial x(t)}=\frac{\partial \delta u}{\partial x(0)} \frac{\partial x(0)}{\partial x(t)}=\delta \boldsymbol{F F}_{0}(t)^{-1}
$$

As for Eq. (18), by using Eq. (16), $\boldsymbol{\delta} \boldsymbol{F}$, the variation of $\boldsymbol{F}$ associate with the virtual displacement $\overrightarrow{\delta u}$, can be identified as:

$$
\boldsymbol{\delta} \boldsymbol{F}=\frac{1}{2 A_{0}}\left(\begin{array}{cc}
\sum_{i=0}^{3} a_{i} \delta u_{i} & \sum_{i=0}^{3} b_{i} \delta u_{i} \\
\sum_{i=0}^{3} a_{i} \delta v_{i} & \sum_{i=0}^{3} b_{i} \delta v_{i}
\end{array}\right)
$$

with $\delta u_{i}, \delta v_{i}$ the virtual $X$ and $Y$ nodal displacements, respectively. The second term of Eq. (21) is then developed in order to identify nodal forces. As previously, $\overrightarrow{\delta u}$ can be identified thanks to Eq. (16) in:

$$
\overrightarrow{\delta u}=\left(\begin{array}{c}
N_{1} \delta u_{1}+N_{2} \delta u_{2}+N_{3} \delta u_{3} \\
N_{1} \delta v_{1}+N_{2} \delta v_{2}+N_{3} \delta v_{3}
\end{array}\right)
$$

Defining $\delta \overrightarrow{u^{N}}$ and $\overrightarrow{P^{N}}$ respectively the nodal displacement vector and the nodal force vector, expressed as:

$$
\delta \overrightarrow{u^{N}}=\left(\begin{array}{c}
\delta u_{1} \\
\delta v_{1} \\
\delta u_{2} \\
\delta v_{2} \\
\delta u_{3} \\
\delta v_{3}
\end{array}\right), \overrightarrow{P^{N}}=\left(\begin{array}{c}
\int_{\Omega S} S_{X} N_{1} d l \\
\int_{\Omega S} S_{Y} N_{1} d l \\
\int_{\Omega S} S_{X} N_{2} d l \\
\int_{\Omega S} S_{Y} N_{2} d l \\
\int_{\Omega S} S_{X} N_{3} d l \\
\int_{\Omega S} S_{Y} N_{3} d l
\end{array}\right),
$$

and assuming stress and deformation gradient constant on the element, it leads that Eq. (21) is equivalent to:

$$
\operatorname{tr}\left(\boldsymbol{\delta} \boldsymbol{F} \boldsymbol{F}_{0}^{-1}(t) \boldsymbol{\sigma}\right) A-\delta \overrightarrow{u^{N}} \cdot \overrightarrow{P^{N}}=0
$$

with $A$ the area of the current (deformed) element. Using the fact that this relation is true for every virtual displacement, the components of $\overrightarrow{P^{N}}$ can be directly identified by splitting the equality with respect to the $\overrightarrow{\delta u}$ components, leading to an equality for each $\vec{P}^{N}$ component.

[1] N. Mohandas and P. G. Gallagher, Red cell membrane: past, present, and future, Blood 112, 3939 (2008).

[2] E. Evans, N. Mohandas, and A. Leung, Static and dynamic rigidities of normal and sickle erythrocytes. major influence of cell hemoglobin concentration, J. Clin. Inv. 73, 477 (1984).

[3] E. A. Evans, Bending elastic modulus of red blood cell membrane derived from buckling instability in micropipet aspiration tests, Biophys. J. 43, 27 (1983). 
[4] M. Abkarian and A. Viallat, Fluid-structure interactions in low-Reynolds-number flows (Royal Society of Chemistry, 2016) Chap. On the importance of red blood cells deformability in blood flow, pp. 347-462.

[5] L. Lanotte, J. Mauer, S. Mendez, D. A. Fedosov, J.-M. Fromental, V. Claveria, F. Nicoud, G. Gompper, and M. Abkarian, Red cells' dynamic morphologies govern blood shear thinning under microcirculatory flow conditions, Proc. Natl Acad. Sc. USA 113, 13289 (2016).

[6] J. Mauer, S. Mendez, L. Lanotte, F. Nicoud, M. Abkarian, G. Gompper, and D. A. Fedosov, Flow-induced transitions of red blood cell shapes under shear, Phys. Rev. Lett. 121 (2018).

[7] R. M. Hochmuth, P. R. Worthy, and E. A. Evans, Red cell extensional recovery and the determination of membrane viscosity, Biophys. J. 26, 101 (1979).

[8] R. M. Hochmuth and R. E. Waugh, Erythrocyte membrane elasticity and viscosity, Ann. Rev. of Phys. 49, 209 (1987).

[9] M. Puig-de Morales-Marinkovic, K. T. Turner, J. P. Butler, J. J. Fredberg, and S. Suresh, Viscoelasticity of the human red blood cell, Am. J. Phys. 293, 597 (2007).

[10] D. A. Fedosov, W. Pan, B. Caswell, G. Gompper, and G. E. Karniadakis, Predicting human blood viscosity in silico, Proc. Natl Acad. Sc. USA 108, 11772 (2011).

[11] J. B. Freund, Numerical simulation of flowing blood cells, Ann. Rev. Fluid Mech. 46, 67 (2014).

[12] P. Balogh and P. Bagchi, Direct numerical simulation of cellular-scale blood flow in 3d microvascular networks, Biophys. J. 113, 2815 (2017).

[13] D. A. Fedosov, B. Caswell, and G. E. Karniadakis, A multiscale red blood cell model with accurate mechanics, rheology, and dynamics, Biophys. J. 98, 2215 (2010).

[14] A. Z. K. Yazdani and P. Bagchi, Influence of membrane viscosity on capsule dynamics in shear flow, J. Fluid Mech. 718, 569 (2013).

[15] Z. Peng, A. Mashayekh, and Q. Zhu, Erythrocyte responses in low-shear-rate flows: effects of non-biconcave stressfree state in the cytoskeleton, J. Fluid Mech. 742, 96 (2014).

[16] D. Cordasco and P. Bagchi, On the shape memory of red blood cells, Phys. Fluids 29, 10.1063/1.4979271 (2017).

[17] F. Guglietta, M. Behr, L. Biferale, G. Falcucci, and M. Sbragaglia, On the effect of membrane viscositity on transient red blood cell dynamics, Soft Mat. 16, 6191 (2020).

[18] W. R. Dodson III and P. Dimitrakopoulos, Tank-treading of erythrocytes in strong shear flows via a nonstiff cytoskeleton-based continuum computational modeling, Biophys. J. 99, 2906 (2010).

[19] S. Mendez and M. Abkarian, Dynamics of blood cell suspensions in microflows (CRC Press, 2019) Chap. Single Red Blood Cell Dynamics in Shear Flow and their Role in Hemorheology.

[20] S. R. Keller and R. Skalak, Motion of a tank-treading ellipsoidal particle in a shear flow, J. Fluid Mech. 120, 27 (1982).

[21] M. Abkarian, M. Faivre, and A. Viallat, Swinging of red blood cells under shear flow, Phys. Rev. Lett. 98, 10.1103/PhysRevLett.98.188302 (2007).

[22] J. M. Skotheim and T. W. Secomb, Red blood cells and other nonspherical capsules in shear flow: Oscillatory dynamics and the tank-treading-to-tumbling transition, Phys. Rev. Lett. 98, 10.1103/PhysRevLett.98.078301 (2007).

[23] J. Dupire, M. Abkarian, and A. Viallat, A simple model to understand the effect of membrane shear elasticity and stress-free shape on the motion of red blood cells in shear flow, Soft Mat. 11, 8372 (2015).

[24] S. Mendez and M. Abkarian, In-plane elasticity controls the full dynamics of red blood cells in shear flow, Phys. Rev. Fluids 3 (2018).

[25] J. E. Mancuso and W. D. Ristenpart, Stretching of red blood cells at high strain rates, Phys. Rev. Fluids 10.1103/PhysRevFluids.2.101101 (2017).

[26] T. M. Fischer, M. Stöhr-Liesen, and H. Schmid-Schönbein, The red cell as a fluid droplet: Tank tread-like motion of the human erythrocyte membrane in shear flow, Science 202, 894 (1978).

[27] T. M. Fischer, Tank-tread frequency of the red cell membrane: Dependence on the viscosity of the suspending medium, Biophys. J. 93, 2553 (2007).

[28] D. Cordasco, Yazdani, and P. Bagchi, Comparison of erythrocyte dynamics in shear flow under different stress-free configurations, Phys. Fluids 26, 10.1063/1.4871300 (2014).

[29] W. R. Dodson III and P. Dimitrakopoulos, Oscillatory tank-treading motion of erythrocytes in shear flows, Phys. Rev. E 84 (2011).

[30] Z. Peng, X. Li, I. V. Pivkin, M. Dao, G. E. Karniadakis, and S. Suresh, Lipid bilayer and cytoskeletal interactions in a red blood cell, Proc. Natl Acad. Sc. USA 110, 13356 (2013).

[31] Z. Peng and Q. Zhu, Deformation of the erythrocyte cytoskeleton in tank treading motions, Soft Mat. 9 (2013).

[32] Z. Peng, S. Salehyar, and Q. Zhu, Stability of the tank treading modes of erythrocytes and its dependence on cytoskeleton reference states, J. Fluid Mech. 771, 449 (2015).

[33] A. Z. K. Yazdani, R. M. Kalluri, and P. Bagchi, Tank-treading and tumbling frequencies of capsules and red blood cells, Phys. Rev. E 83 (2011).

[34] T. M. Fischer, On the energy dissipation in a tank-treading human red blood cell, Biophys. J. 32, 863 (1980).

[35] R. Tran-Son-Tay, S. P. Sutera, and P. R. Rao, Determination of red blood cell membrane viscosity from rheoscopic observations of tank-treading motion, Biophys. J. 46, 65 (1984).

[36] C. D. Eggleton and A. S. Popel, Large deformation of red blood cell ghosts in a simple shear flow, Phys. Fluids 10, 1834 (1998).

[37] C. S. Peskin, The immersed boundary method, Acta Num. 11, 479 (2002).

[38] P. Li and J. Zhang, A finte difference method with subsampling for immersed boundary simulations of the capsule dynamics with viscoelastic membranes, Int. J. Numer. Meth. Eng. 35, 10.1002/cnm.3200 (2019). 
[39] J. C. Simo, On a fully three-dimensional finite-strain viscoelastic damage model: Formulation and computational aspects, Comput. Meth. Appl. Mech. Eng. 60, 153 (1887).

[40] R. Skalak, A. Tozeren, R. P. Zarda, and S. Chien, Strain energy function of red blood cell membranes, Biophys. J. 13, 245 (1973).

[41] P. Bagchi and A. Z. K. Yazdani, Analysis of membrane tank-tread of nonspherical capsules and red blood cells, Eur. Phys. J. E 35 (2012).

[42] Z. Peng, R. J. Asaro, and Q. Zhu, Multiscale modelling of erythrocytes in Stokes flow, J. Fluid Mech. 686, 299 (2011).

[43] E. A. Evans, New membrane concept applied to the analysis of fluid shear- and micropipette-deformed red blood cells, Biophys. J. 13, 941 (1973).

[44] M. Dao, C. T. Lim, and S. Suresh, Mechanics of the human red blood cell deformed by optical tweezers, J. Mech. Phys. Sol. 51, 2259 (2003).

[45] J. Sigüenza, S. Mendez, and F. Nicoud, How should the optical tweezers experiment be used to characterize the red blood cell membrane mechanics?, Biomech. Model. Mechanobiol. 16, 1645 (2017).

[46] P. Taraconat, J.-P. Gineys, D. Isèbe, F. Nicoud, and S. Mendez, Numerical simulation of deformable particles in a Coulter counter, Int. J. Numer. Meth. Biomed. Eng. 35, e3243 (2019).

[47] A. Chorin, Numerical solution of the Navier-Stokes equations, Math. Comp. 22, 745 (1968).

[48] V. Moureau, P. Domingo, and L. Vervisch, Design of a massively parallel CFD code for complex geometries, Comp. Rend. Méc. 339, 141 (2011).

[49] M. Malandain, N. Maheu, and V. Moureau, Optimization of the deflated conjugate gradient algorithm for the solving of elliptic equations on massively parallel machines, J. Comput. Phys. 238, 32 (2013).

[50] S. Mendez, E. Gibaud, and F. Nicoud, An unstructured solver for simulations of deformable particles in flows at arbitrary Reynolds numbers, J. Comput. Phys. 256, 465 (2014).

[51] J. Sigüenza, S. Mendez, D. Ambard, F. Dubois, F. Jourdan, R. Mozul, and F. Nicoud, Validation of an immersed thick boundary method for simulating fluid-structure interactions of deformable membranes, J. Comput. Phys. 322, 723 (2016).

[52] D. Cordasco and P. Bagchi, Orbital drift of capsules and red blood cells in shear flow, Phys. Fluids 25, 10.1063/1.4820472 (2013).

[53] D. Cordasco and P. Bagchi, Intermittency and synchronized motion of red blood cell dynamics in shear flow, J. Fluid Mech. 759, 472 (2014).

[54] J.-M. Charrier, S. Shrivastava, and R. Wu, Free and constrained inflation of elastic membranes in relation to thermoforming non-axisymmetric problems, J. Strain Anal. Eng. Des. 24, 55 (1989).

[55] S. Shrivastava and J. Tang, Large deformation finite element analysis of non-linear viscoelastic membranes with reference to thermoforming, J. Strain Anal. Eng. Des. 28, 31 (1993).

[56] S. Chien, Shear dependence of effective cell volume as a determinant of blood viscosity, Science 168, 977 (1970).

[57] K. Monkos and B. Turczynski, Determination of the axial ratio of globular proteins in aqueous solution using viscometric measurements, Int. J. Bio. Macro. (1991).

[58] K. Monkos, Viscometric study of human, bovine, equine and ovine haemoglobin in aqueous solution, Int. J. Bio. Macro. 16, 31 (1994).

[59] G. Lenormand, S. Hénon, A. Richet, J. Siméon, and F. Gallet, Direct measurement of the area expansion and shear moduli of the human red blood cell membrane skeleton, Biophys. J. 81, 43 (2001).

[60] A. Pinelli, I. Z. Naqavi, U. Piomelli, and J. Favier, Immersed-boundary methods for general finite-difference and finite-volume Navier-Stokes solvers, J. Comput. Phys. 229, 9073 (2010).

[61] A. Bogdanova, L. Kaestner, G. Simionato, A. Wickrema, and A. Makhro, Heterogeneity of red blood cells: Causes and consequences, Front. Physiol. 11, 1 (2020).

[62] J. Dupire, M. Socol, and A. Viallat, Full dynamics of a red blood cell in shear flow, Proc. Natl Acad. Sc. USA 109, 20808 (2012).

[63] S. Chien, K.-L. P. Sung, R. Skalak, S. Usami, and Tözeren, Red cell extensional recovery and the determination of membrane viscosity, Biophys. J. 24, 463 (1978).

[64] S. Hénon, A new determination of the shear modulus of the human erythrocyte membrane using optical tweezers, Biophys. J. 76, 1145 (1999).

[65] D. Arora, M. Behr, and M. Pasquali, A tensor-based measure for estimating blood damage, Artif. Organs 28, 1002 (2004). 